\title{
Short communication: Probiotic induction of interleukin-10 and interleukin-12 production by macrophages is modulated by co-stimulation with microbial components
}

\author{
Rumi Kaji, Junko Kiyoshima-Shibata, Satoshi Tsujibe, Masanobu Nanno, and Kan Shida ${ }^{1}$ \\ Yakult Central Institute, 5-11 Izumi, Kunitachi-shi, Tokyo 186-8650, Japan
}

\begin{abstract}
Probiotic lactobacilli stimulate macrophages and dendritic cells to secrete cytokines and thereby regulate the immune responses of the host. The balance of the IL-10 and IL-12 production induced by a probiotic is crucial for determining the direction of the immune response. In the present study, we examined the ability of microbial components to modify IL-10 and IL-12 production induced by a popular probiotic strain, Lactobacillus casei strain Shirota ( $\mathrm{LcS}$ ), which itself predominantly induces IL-12 production. Microbial ligands for toll-like receptor (TLR) 3 and TLR5 further enhanced the IL-12 induction by LcS, whereas ligands for TLR2, TLR4, TLR7, and TLR9 converted the cytokine production pattern from IL-12 predominant to IL-10 predominant. These results indicate that the probiotic induction of IL-10 and IL-12 production can be flexibly modified by co-stimulation with microbial components. This could explain the variety of immunomodulatory functions (immunoactivation or anti-inflammation) exerted by this probiotic strain.
\end{abstract}

Key words: probiotic, interleukin-10, interleukin-12, toll-like receptor, macrophage

\section{Short Communication}

Probiotics are live microorganisms that exert health benefits in the host, with currently popular probiotics including some strains of lactobacilli (WHO/FAO, 2006). One of the most attractive health benefits of probiotics is immune modulation. Thus, several strains of probiotics have been used to make functional dairy foods that aim to maintain the immunological homeostasis of the host (van Baarlen et al., 2013; Linares et al., 2017).

Ingested probiotics must encounter macrophages and dendritic cells to induce the production of a range of

Received September 21, 2017.

Accepted December 14, 2017.

${ }^{1}$ Corresponding author: kan-shida@yakult.co.jp cytokines, which themselves trigger various immune responses. In particular, the cytokines IL-12 and IL-10 have received special attention because IL-12 is key to augmenting the immune defense against infections and cancers, whereas IL-10 is critical for regulating excessive immune responses to avoid inflammatory diseases (Trinchieri, 2003; Ouyang et al., 2011). The production of the 2 cytokines is reciprocally regulated (Ma et al., 2015), and the IL-10 and IL-12 response varies with each strain of probiotics, which may be responsible for their specific effects on the host immune system (Kaji et al., 2010; Rask et al., 2013).

We previously elucidated that Lactobacillus casei strain Shirota $(\mathbf{L c S})$, one of the most popular probiotic strains with immunomodulatory activities, can induce a large amount of IL-12 and little IL-10 and that stimulation of macrophages via the 3-dimensional structure of the cell wall is critical for their cytokine-inducing activity (Shida et al., 2006). In addition, the cell wall teichoic acid derived from Lactobacillus plantarum can convert the predominant IL-12 response induced by LcS into a predominant IL-10 response via toll-like receptor (TLR)2-dependent extracellular signal-regulated kinase (ERK) activation in macrophages (Kaji et al., 2010). Modification of the IL-10 and IL-12 response by TLR-mediated stimuli has also been reported in dendritic cells and monocytes (Barkman et al., 2008; Baba et al., 2009). Thus, we hypothesized that some microbial components other than TLR2 ligands might alter the original properties of the IL-12-inducing immunostimulatory LcS. This would help to explain the variety of its clinical efficacies mediated through immune modulation (Shida et al., 2011). In the present study, we examined the modifying effect of various TLR ligands on IL-10 and IL-12 production by using macrophages stimulated with LcS.

Highly purified or chemically synthesized microbial components/mimics and killed lactobacillus cells were used in this study to understand the basic mechanism of the flexible cytokine production induced by probiotics. The following TLR ligands were purchased from InvivoGen (San Diego, CA): lipoteichoic acid (LTA; 
TLR2 ligand), poly (I:C) (TLR3 ligand), LPS (TLR4 ligand), flagellin (TLR5 ligand), gardiquimod (TLR7 ligand), and CpG-DNA (TLR9 ligand). Heat-killed LcS (YIT 9029) and Lactobacillus johnsonii YIT $0219^{\mathrm{T}}$ were prepared as described previously (Shida et al., 2006). Briefly, LcS and L. johnsonii were obtained from the culture collection of the Yakult Central Institute (Tokyo, Japan), cultured for $20 \mathrm{~h}$ at $37^{\circ} \mathrm{C}$ in Lactobacilli-de Man, Rogosa, and Sharpe broth (Difco, Detroit, MI), collected by centrifugation, heated at $100^{\circ} \mathrm{C}$ for $30 \mathrm{~min}$, and then lyophilized. Peritoneal macrophages were prepared from BALB/c mice (Japan Clea Co., Tokyo, Japan) $4 \mathrm{~d}$ after intraperitoneal injection of $4 \%$ thioglycollate broth (Difco), as described previously (Shida et al., 2006). Mice were used at 8 to $12 \mathrm{wk}$ of age in accordance with the guidelines for the care and use of laboratory animals established by the Yakult Central Institute.

To test the response of IL-10 and IL-12 production, macrophages $\left(1 \times 10^{5}\right.$ cells $)$ were cultured with each TLR ligand $(1 \mu \mathrm{g} / \mathrm{mL})$ in the presence or absence of heat-killed lactobacilli $(10 \mu \mathrm{g} / \mathrm{mL})$ in $0.2 \mathrm{~mL}$ RPMI 1640 medium containing $10 \%$ fetal calf serum in a 96-well culture plate for $24 \mathrm{~h}$. The supernatants were collected, and IL-10 and IL-12p70 levels were analyzed by ELISA. Rat anti-mouse IL-12p35 (clone 9A5) and biotinylated rat anti-mouse IL-12p40 (clone C17.8) monoclonal antibodies were used as capture and detection antibodies, respectively. The antibodies and recombinant mouse IL-12p70 were purchased from BD Pharmingen (San Diego, CA). The mouse IL-10 OptEIA set (BD Bioscience, San Diego, CA) was used to determine IL-10 concentrations.

As shown in Figure 1, TLR ligands alone did not induce substantial levels of IL-12 production, in accordance with previous reports that soluble bacterial cellular components can barely induce IL-12 production in macrophages and monocytes (Shida et al., 2006; Barkman et al., 2008). The LTA, LPS, gardiquimod, and CpG-DNA alone induced substantial levels of IL-10. The LcS alone strongly induced IL-12 production but only weakly induced IL-10 production, indicating that this strain is a potent IL-12 inducer. In combination with LcS, the ligands for TLR3 [poly (I:C)] and TLR5 (flagellin) further enhanced IL-12 production induced by LcS. In contrast, the ligands for TLR2 (LTA), TLR4 (LPS), TLR7 (gardiquimod), and TLR9 (CpG-DNA) synergistically enhanced IL-10 production with LcS but inhibited IL-12 production induced by LcS alone. We previously showed that predominant IL-12 production induced by LcS can be converted to predominant IL10 production by co-stimulation with a TLR2 ligand, cell wall teichoic acid (Kaji et al., 2010). The present data indicate that some TLR ligands other than TLR2

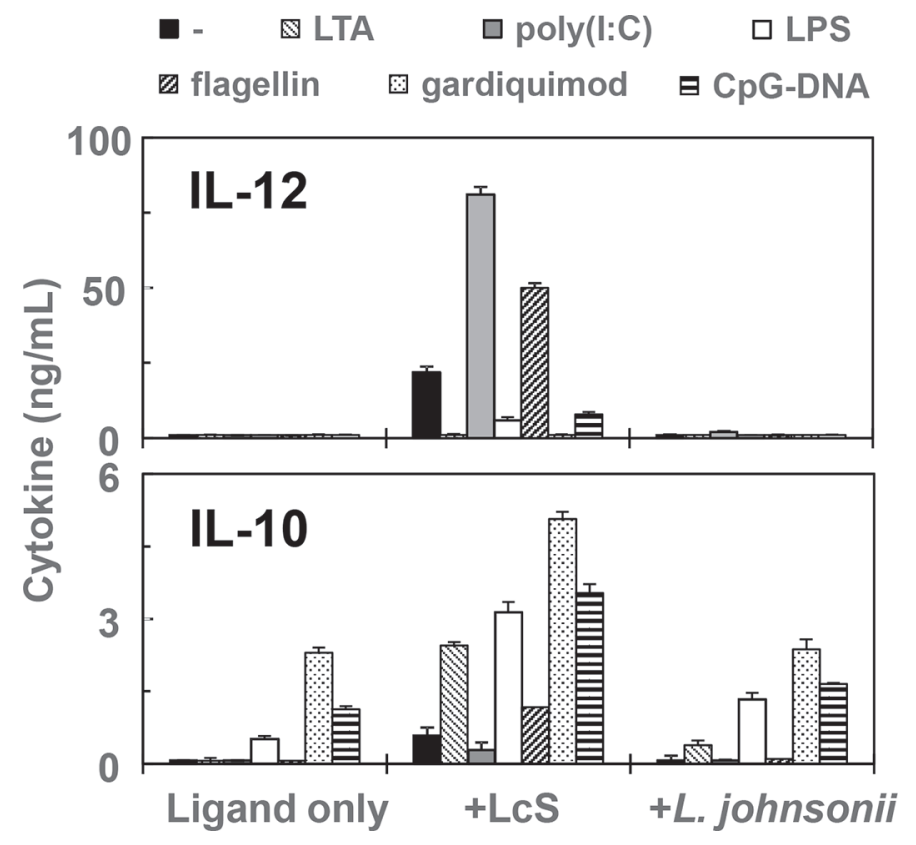

Figure 1. Modification by toll-like receptor (TLR) ligands of IL10 and IL-12 production induced by Lactobacillus casei strain Shirota $(\mathrm{LcS})$. Peritoneal macrophages were cultured with TLR ligands (1 $\mu \mathrm{g} / \mathrm{mL}$ ) in the presence or absence of lactobacilli (LcS, Lactobacillus johnsonii; $10 \mu \mathrm{g} / \mathrm{mL}$ ) for $24 \mathrm{~h}$, and the levels of IL-10 and IL-12 in supernatants were determined by ELISA. Data are the mean \pm SD of triplicate cultures. Experiments were repeated twice with similar results. $-=$ medium only; LTA = lipoteichoic acid; poly(I:C) = polyinosinic: polycytidylic acid; CpG-DNA = DNA containing unmethylated cytosine-phosphate-guanine motifs.

ligands can also alter the balance of IL-10 and IL-12 production induced by LcS from IL-12 predominance to IL-10 predominance.

The modulatory effects of TLR ligands on cytokine production were not observed in the case of co-stimulation with $L$. johnsonii, which originally induced very low levels of IL-10 and IL-12 (Figure 1). We previously showed that L. johnsonii, unlike LcS, is digested rapidly in macrophages after phagocytosis and cannot stimulate them via the 3-dimensional structure of the cell wall (Shida et al., 2006). It may be possible that TLR-derived signals modulate only the signals derived from the cell wall structure to lead to flexible IL-10 and IL-12 production.

We previously determined that activation of the ERK pathway via TLR2 is crucial for the conversion of the IL-10 and IL-12 balance from predominantly IL-12 to predominantly IL-10 in LcS-stimulated macrophages (Kaji et al., 2010). Thus, we next examined ERK activation induced by TLR ligands in macrophages. Macrophages $\left(1 \times 10^{6}\right.$ cells $)$ were cultured with each TLR ligand $(1 \mu \mathrm{g} / \mathrm{mL})$ in $2 \mathrm{~mL}$ of RPMI 1640 medium containing $10 \%$ fetal calf serum in a 24 -well culture plate for $0.5,1$, and $2 \mathrm{~h}$. The cells were lysed with $1 \%$ Non- 
idet $\mathrm{P}-40$ in $25 \mathrm{~m} M$ Tris-HCl buffer (pH 7.5) and used for analysis of ERK activation, as described previously (Kaji et al., 2010). Briefly, the lysates of the stimulated cells were separated by SDS-PAGE, transferred onto a polyvinylidene difluoride membrane (Pall Corp., East Hills, NY), and then analyzed for phosphorylated and total ERK by immunoblotting using specific antibodies to phosphorylated and total ERK (Cell Signaling Technology, Beverly, MA).

As shown in Figure 2, the ligands for TLR2, TLR4, and TLR7 strongly activated the ERK pathway, whereas the ligands for TLR3 and TLR5 had little effect on phosphorylated ERK levels. The data showing that the ligands for TLR2, TLR4, and TLR7, but not TLR3 and TLR5, induced strong activation of ERK and then converted the IL-10 and IL-12 balance to IL-10 predominance is in accordance with our previous findings (Kaji et al., 2010). However, CpG-DNA (TLR9 ligand), which changed the IL-10 and IL-12 balance to IL-10 predominance, had a negligible effect on ERK activation, similar to the ligands for TLR3 and TLR5. The CpG-DNA seems to be an exception to the rule that TLR ligands that strongly activate the ERK pathway convert the IL-10 and IL-12 balance from IL12 predominance to IL-10 predominance, indicating that activation of the ERK pathway is not necessary to shift the balance from IL-12 predominance to IL10 predominance in certain cases. Although it is not clear whether the exception is applicable to only CpGDNA or all potential TLR9 ligands, CpG-DNA has at least been shown to induce IL-10 production through an ERK-independent pathway in bone marrow-derived macrophages (Banerjee et al., 2006).

Ligands for TLR3 and TLR5, in contrast to those for the other TLR, further enhanced IL-12 production induced by LcS. Similarly, TLR3 ligand enhanced $L$. casei-induced IL-12 production in monocyte-derived dendritic cells (Baba et al., 2009). The TLR3 uses a different downstream signaling pathway from the other TLR, the TRIF pathway (Takeda and Akira, 2005). However, no common signaling pathway has been found for TLR3 and TLR5. Although the molecular mechanism by which the 2 TLR stimuli lead to a different cytokine modulation pattern from that induced by TLR2, TLR4, TLR7, and TLR9 cannot be explained, this aspect is of particular interest from a biological point of view. Because ligands for TLR3 and TLR5 are commonly found in pathogenic viruses and bacteria (Takeda and Akira, 2005), LcS would induce a greater amount of IL-12 in the presence of co-stimulation with such pathogenic microbial components to further augment the host defense (Figure 3). On the other hand, because ligands for TLR2, TLR4, and TLR9 are widely present in numerous nonpathogenic commensal bacte-

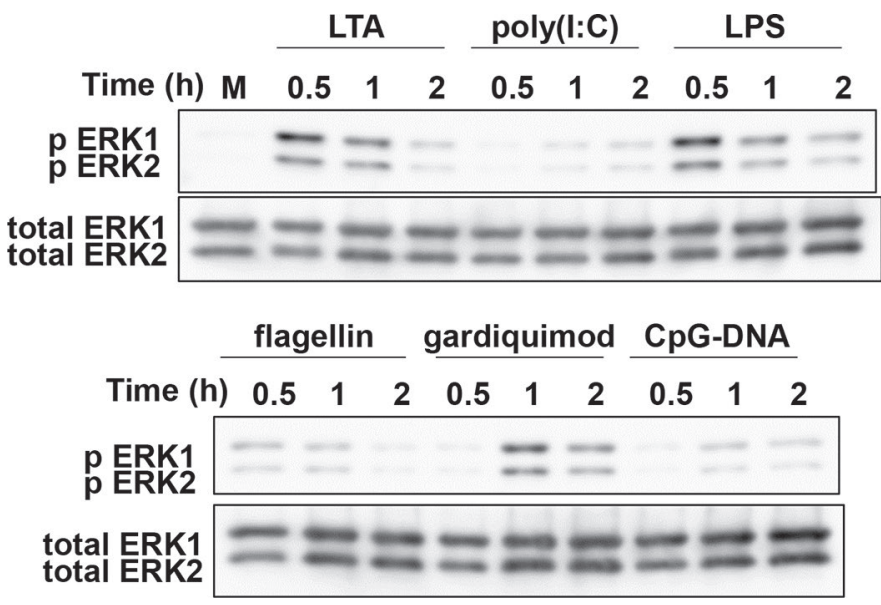

Figure 2. Extracellular signal-regulated kinase (ERK) activation in macrophages by stimulation with toll-like receptor (TLR) ligands. Peritoneal macrophages were cultured with TLR ligands $(1 \mu \mathrm{g} / \mathrm{mL})$ for $0.5,1$, and $2 \mathrm{~h}$. Cell lysates were prepared and analyzed for ERK activation by immunoblotting using specific antibodies to phosphorylated $(\mathrm{p})$ and total ERK. Experiments were repeated twice with similar results. $\mathrm{M}=$ unstimulated; LTA = lipoteichoic acid; poly $(\mathrm{I}: \mathrm{C})=$ polyinosinic: polycytidylic acid; CpG-DNA $=$ DNA containing unmethylated cytosine-phosphate-guanine motifs.

ria (Takeda and Akira, 2005), LcS may be likely to induce IL-10, rather than IL-12, in collaboration with the bacteria at such sites as homeostatic intestinal mucosa.

Several health-promoting, immunomodulatory activities of LcS have been identified in human clinical

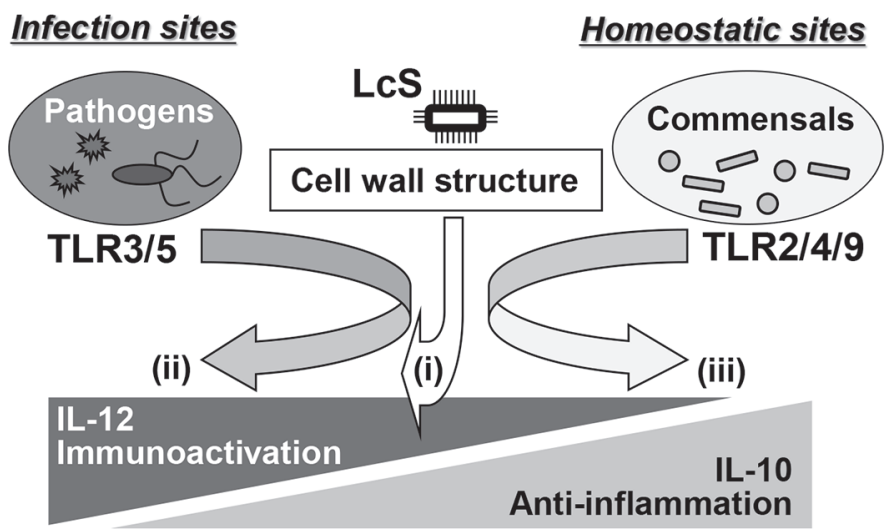

Figure 3. Proposed model of the dual immunomodulation of Lactobacillus casei strain Shirota (LcS) in collaboration with coexisting microbes. The $\mathrm{LcS}$ alone stimulates macrophages via the 3-dimensional structure of their cell wall to induce predominant IL-12 production (i). The LcS would more strongly induce IL-12 production in collaboration with pathogen-derived toll-like receptor (TLR) 3 and TLR5 ligands to augment host defense at infection sites (ii), whereas it would induce predominant IL-10 production in collaboration with nonpathogenic commensal bacterium-derived TLR2, TLR4, and TLR9 ligands at intestinal mucosal sites (iii). The induced IL-10 would help to maintain physiological inflammation under homeostatic conditions and also to downregulate pathological inflammation in the case of inflammatory bowel diseases. 
trials and experimental animal models. For example, administration of LcS restored natural killer cell activity and augmented the host defense mechanism (Shida and Nomoto, 2013; Shida et al., 2017). The LcS also improved some autoimmune diseases and inflammatory bowel diseases, indicating that this strain suppresses excessive immune responses (Matsuzaki et al., 1997; Mitsuyama et al., 2008; Lei et al., 2017). Therefore, LcS is likely to play dual roles in immune regulation (immunoactivation and anti-inflammation) and the IL-12 and IL-10 induced by LcS are suggested to participate in such activities (Matsuzaki et al., 1997; Shida and Nomoto, 2013). The present observation that LcS-induced IL-10 and IL-12 production can be flexibly modified by microbial ligands for TLR would help to explain the variety of the immunomodulatory activities of LcS.

The effectiveness of a given probiotic in immunomodulation often differs among individuals. Here, we show that certain probiotics can flexibly regulate cytokine responses according to the microbial environment. The present findings might help to explain the individual differences in probiotic effectiveness, considering that individual-specific gut microbiota would provide corresponding bystander immune modulators, namely, TLR ligands.

\section{ACKNOWLEDGMENTS}

We thank the staff of the animal facility of the Yakult Central Institute for their expertise in mouse breeding. This study was funded by the Yakult Honsha Co. Ltd. (Tokyo, Japan), which produces and markets fermented milk beverages with LcS.

\section{REFERENCES}

Baba, N., S. Samson, R. Bourdet-Sicard, M. Rubio, and M. Sarfati. 2009. Selected commensal-related bacteria and Toll-like receptor 3 agonist combinatorial codes synergistically induce interleukin- 12 production by dendritic cells to trigger a $\mathrm{T}$ helper type 1 polarizing programme. Immunology 128:e523-e531.

Banerjee, A., R. Gugasyan, M. McMahon, and S. Gerondakis. 2006. Diverse Toll-like receptors utilize $\mathrm{Tpl} 2$ to activate extracellular signal-regulated kinase (ERK) in hemopoietic cells. Proc. Natl. Acad. Sci. USA 103:3274-3279.

Barkman, C., A. Martner, C. Hessle, and A. E. Wold. 2008. Soluble bacterial constituents down-regulate secretion of IL-12 in response to intact Gram-positive bacteria. Microbes Infect. 10:1484-1493.
Kaji, R., J. Kiyoshima-Shibata, M. Nagaoka, M. Nanno, and K. Shida. 2010. Bacterial teichoic acids reverse predominant IL-12 production induced by certain lactobacillus strains into predominant IL-10 production via TLR2-dependent ERK activation in macrophages. J. Immunol. 184:3505-3513.

Lei, M., C. Guo, D. Wang, C. Zhang, and L. Hua. 2017. The effect of probiotic Lactobacillus casei Shirota on knee osteoarthritis: A randomised double-blind, placebo-controlled clinical trial. Benef. Microbes 8:697-703.

Linares, D. M., C. Gomez, E. Renes, J. M. Fresno, M. E. Tornadijo, R. P. Ross, and C. Stanton. 2017. Lactic acid bacteria and bifidobacteria with potential to design natural biofunctional healthpromoting dairy foods. Front. Microbiol. 8:846.

Ma, X., W. Yan, H. Zheng, Q. Du, L. Zhang, Y. Ban, N. Li, and F. Wei. 2015. Regulation of IL-10 and IL-12 production and function in macrophages and dendritic cells. F1000Res. 4. pii: F1000 Faculty Rev-1465. eCollection 2015. https://doi.org/10.12688/ f1000research.7010.1.

Matsuzaki, T., Y. Nagata, S. Kado, K. Uchida, I. Kato, S. Hashimoto, and T. Yokokura. 1997. Prevention of onset in an insulindependent diabetes mellitus model, NOD mice, by oral feeding of Lactobacillus casei. APMIS 105:643-649.

Mitsuyama, K., S. Matsumoto, H. Yamasaki, J. Masuda, K. Kuwaki, H. Takedatsu, M. Nagaoka, A. Andoh, O. Tsuruta, and M. Sata. 2008. Beneficial effects of Lactobacillus casei in ulcerative colitis: A pilot study. J. Clin. Biochem. Nutr. 43:s78-s81.

Ouyang, W., S. Rutz, N. K. Crellin, P. A. Valdez, and S. G. Hymowitz. 2011. Regulation and functions of the IL-10 family of cytokines in inflammation and disease. Annu. Rev. Immunol. 29:71-109.

Rask, C., I. Adlerberth, A. Berggren, I. L. Ahren, and A. E. Wold. 2013. Differential effect on cell-mediated immunity in human volunteers after intake of different lactobacilli. Clin. Exp. Immunol. 172:321-332.

Shida, K., J. Kiyoshima-Shibata, M. Nagaoka, K. Watanabe, and M. Nanno. 2006. Induction of interleukin-12 by Lactobacillus strains having a rigid cell wall resistant to intracellular digestion. J. Dairy Sci. 89:3306-3317.

Shida, K., M. Nanno, and S. Nagata. 2011. Flexible cytokine production by macrophages and $\mathrm{T}$ cells in response to probiotic bacteria: A possible mechanism by which probiotics exert multifunctional immune regulatory activities. Gut Microbes 2:109-114.

Shida, K., and K. Nomoto. 2013. Probiotics as efficient immunopotentiators: Translational role in cancer prevention. Indian J. Med. Res. 138:808-814.

Shida, K., T. Sato, R. Iizuka, R. Hoshi, O. Watanabe, T. Igarashi, K. Miyazaki, M. Nanno, and F. Ishikawa. 2017. Daily intake of fermented milk with Lactobacillus casei strain Shirota reduces the incidence and duration of upper respiratory tract infections in healthy middle-aged office workers. Eur. J. Nutr. 56:45-53.

Takeda, K., and S. Akira. 2005. Toll-like receptors in innate immunity. Int. Immunol. 17:1-14.

Trinchieri, G. 2003. Interleukin-12 and the regulation of innate resistance and adaptive immunity. Nat. Rev. Immunol. 3:133-146.

van Baarlen, P., J. M. Wells, and M. Kleerebezem. 2013. Regulation of intestinal homeostasis and immunity with probiotic lactobacilli. Trends Immunol. 34:208-215.

WHO and FAO. 2006. FAO food and nutrition paper 85. Probiotics in food: Health and nutritional properties and guidelines for evaluation. Accessed Jan. 19, 2018. http://www.fao.org/3/a-a0512e.pdf. 